\title{
Periodontal treatment did not prevent complications of pregnancy
}

\author{
Abstracted from \\ Newnham JP, Newnham IA, Ball CM, et al. \\ Treatment of periodontal disease during pregnancy: a randomised controlled trial. \\ Obstet Gynecol 2009; 114: 1239-1248 \\ Address for correspondence: John P Newnham, FRANZCOG, School of Women's and Infants' Health, \\ University of Western Australia, King Edward Memorial Hospital, 374 Bagot Road, Subiaco, \\ Perth WA 6008, Australia. E-mail: john.newnham@uwa.edu.au
}

\section{Question: Does the treatment of periodontal disease in midpregnancy prevent preterm birth, foetal growth restriction, and preeclampsia?}

Design A randomised controlled trial (RCT) was conducted. Intervention Women found to have a periodontal disease were randomly allocated to receive periodontal treatment in midpregnancy (this was the treatment group; $\mathrm{n}=542$ ) or after the pregnancy was concluded (the control group; $\mathrm{n}=540$ ). Periodontal disease was defined as presence of periodontal pockets of $4 \mathrm{~mm}$ or greater in depth at 12 or more probing sites in fully erupted teeth (typically excluding wisdom teeth). Treatments were conducted either by the hygienists or periodontists and included nonsurgical debridement of the subgingival and supragingival plaque, removal of local predisposing factors such as calculus, root planing, and adjustment of overhanging restorations. Comprehensive oral hygiene instructions and motivation were provided at each visit at a minimum of three weekly visits, with further visits if required.

Outcome measure The primary outcomes were preterm birth or other major complications of pregnancy.

Results There were no differences between the control and treatment groups in terms of: preterm birth [9.3\% compared with 9.7\%; odds ratio (OR), 1.05; 95\% confidence interval $(\mathrm{Cl}), 0.7-1.58 ; \mathrm{P} 0.81)$; birthweight (3450 g versus $3410 \mathrm{~g}$; P 0.12); pre-eclampsia (4.1\% versus 3.4\%; OR, $0.82 ; 95 \% \mathrm{Cl}, 0.44-1.56$; P 0.55 ); or other obstetric endpoints. There were four unexplained stillbirths in the control group and no pregnancy losses in the treated group (P 0.12). Measures of foetal and neonatal wellbeing were similar in the two groups, including abnormalities in foetal heart rate recordings ( $P$ 0.26), umbilical artery flow studies (P 0.96), and umbilical artery blood gas values (P 0.37). The periodontal treatment was highly successful in improving health of the gums $(P<0.01)$.

Conclusions The evidence provided by the present study does not support the hypothesis that treatment of periodontal disease during pregnancy in this population prevents preterm birth, foetal growth restriction, or pre-eclampsia. Periodontal treatment was not hazardous to the women or their pregnancies.

\section{Commentary}

In their seminal 1996 case-control study of 124 women, Offenbacher and colleagues ${ }^{1}$ postulated that periodontal disease is a risk factor for preterm and low birthweight. The underlying mechanism for this occurrence was postulated to be a Gram-negative bacterial infection of the periodontium.

Like the curses in Chaucer's The Parson's Tale, the well-conducted and -reported study by Newnham et al. (2009) is like, "a bird that returns again to his own nest". This RCT of approximately 1000 women parallels recent large scale RCT by Michalowicz et al. (2006; 800 women $)^{2}$ and Offenbacher et al. (2009; 1800 women). ${ }^{3}$ The data from all three studies point in the same direction: periodontal therapy with scaling and root planing does not reduce the occurrence of adverse pregnancy outcomes.

Is the original Offenbacher paper's hypothesis therefore incorrect? There are at least two watch words brought to mind by this question and the clinical trials: caution and biology.

Let me address caution first. The initial 1996 Offenbacher et al. case-control study provided 'level 3' evidence. This publication created such a stir that over the next 14 years, 195 additional studies were published examining periodontal diseaseobstetric hypothesis (MEDLINE search strategy: "Periodontal Diseases"[Mesh] AND "Obstetric Labor Complications"[Mesh]). Among the 195 reports, almost 50 were narrative reviews supporting the Offenbacher hypothesis.

Seventeen of the 195 reports were RCT, however, ie, 'level 1' evidence. Almost all of the level-1 trials agree in their conclusions taking the opposite view to both the level-3 case-control evidence and the level-5 narrative reviews. Specifically, there is no relationship between periodontal therapy with scaling and root planing and a reduction in preterm or low birthweight.

This dichotomy in the findings is a stark reminder of how powerfully biological concepts can capture one's imagination, in spite of high-level evidence to the contrary.

This brings me fully to the concept of biology, or more specifically the biological concept of the periodontal infection. Almost all the trials refuting the original Offenbacher hypothesis (including Offenbacher's 2009 study) ${ }^{3}$ used scaling and root planing to treat the periodontal infection. Interestingly, there is little or no evidence that this therapy significantly reduces or alters the periodontal microbial infection. In marked contrast, there is very convincing evidence that systemic antibiotics, and specifically short-term metronidazole plus amoxicillin, have a profound, long-term effect on both reducing the bacterial infection and improving periodontal health (eg, Lopez et al., 2006). ${ }^{4}$ 
So, is there really no relationship between periodontal disease and preterm or low birthweight? Or did the scientists just ask the wrong question? Would the clinical trial results have been the same if the clinicians had prescribed systemic metronidazole plus amoxicillin instead of scaling and root planing? We do not know. Given the time and expense invested over the last decade, though, it is unlikely that agencies will invest again in trials examining this question any time soon.

That said, were it me, I would be inclined to treat the periodontal disease with scaling and root planing and with metronidazole plus amoxicillin - and hope that the curses didn't return. After all, the work of Ioannides $(2007)^{5}$ suggests why the Offenbacher hypothesis may in fact be correct, and the clinical trials may be wrong, but for all of the wrong reasons.
Richard Niederman

Centre for Evidence-Based Dentistry, The Forsyth Institute, Boston, Massachusetts, USA

1. Offenbacher $S$, Katz V, Fertik G, et al. Periodontal infection as a possible risk factor for preterm low birth weight. / Periodontol 1996; 67 (suppl.): S1103-S1113.

2. Michalowicz BS, Hodges [S, DiAngelis A], et al. OPT Study. Treatment of periodontal disease and the risk of preterm birth. N Engl J Med 2006; 355: 1885-1894.

3. Offenbacher S, Beck JD, Jared HL, et al. Effects of periodontal therapy on rate of preterm delivery: a randomised controlled trial. Obstet Gynecol 2009; 114: $551-559$.

4. López NJ, Socransky SS, Da Silva I, Japlit MR, Haffajee AD. Effects of metronidazole plus amoxicillin as the only therapy on the microbiological and clinical parameters of untreated chronic periodontitis. / Clin Periodontol 2006; 33: 648-660.

5. Ioannidis JP. Why most published research findings are false. PLoS Med 2005; 2:e124.

Evidence-Based Dentistry (2010) 11, 18-19. doi:10.1038/sj.ebd.6400705 